\title{
AvrXa7-Xa7 Mediated Defense in Rice Can Be Suppressed by Transcriptional Activator-Like Effectors TAL6 and TAL11a from Xanthomonas oryzae pv. oryzicola
}

\author{
Zhi-Yuan Ji, ${ }^{1}$ Li Xiong, ${ }^{1}$ Li-Fang Zou, ${ }^{1}$ Yu-Rong Li, ${ }^{1}$ Wen-Xiu Ma, ${ }^{1}$ Liang Liu, ${ }^{1}$ Muhammad Zakria, ${ }^{1}$ \\ Guang-Hai Ji, ${ }^{2}$ and Gong-You Chen ${ }^{1}$ \\ ${ }^{1}$ School of Agriculture and Biology, Shanghai Jiao Tong University/State Key Laboratory of Microbial Metabolism,, Shanghai, \\ 200240, China; ${ }^{2}$ Key Laboratory for Plant Pathology of Yunnan Province, College of Plant Protection, Yunnan Agricultural \\ University, Kunming, 650201, China
}

Submitted 22 September 2013. Accepted 22 April 2014.

Readers of Ji et al., 27:983-995 (2014) have advised the Editor-in-Chief of several identical images used to represent different experiments. The corresponding author of this paper agrees to be responsible for the misrepresentation of these data and to retract the paper. 